\title{
Weak antiferromagnetic superexchange interaction in fcc $\mathrm{C}_{60} \mathrm{H}_{n}$
}

\author{
Yun-Ki Choi, ${ }^{1}$ Jun-Hyung Cho, ${ }^{1, *}$ Biplab Sanyal, ${ }^{2}$ and Gustav Bihlmayer ${ }^{3}$ \\ ${ }^{1}$ Department of Physics and Research Institute for Natural Sciences, Hanyang University, 17 Haengdang-Dong, \\ Seongdong-Ku, Seoul 133-791, Korea \\ ${ }^{2}$ Division of Materials Theory, Department of Physics and Astronomy, Angstromlaboratoriet, Uppsala University, \\ Box 516, SE-75120, Uppsala, Sweden \\ ${ }^{3}$ Institut für Festkörperforschung and Institute for Advanced Simulation Forschungszentrum Jülich GmbH, D-52425 Jülich, Germany
}

(Received 9 July 2012; published 31 August 2012)

\begin{abstract}
A recent density-functional calculation for fcc $\mathrm{C}_{60} \mathrm{H}_{n}(n=$ odd) [K. W. Lee and C. E. Lee, Phys. Rev. Lett. 106, 166402 (2011)] proposed the existence of Stoner ferromagnetism based on an itinerant band model. However, our density-functional calculation shows that the antiferromagnetic (AFM) configuration is slightly more stable than the ferromagnetic (FM) one. This preference for antiferromagnetism over ferromagnetism is analogous to the case of a dimer $\left(\mathrm{C}_{60} \mathrm{H}\right)_{2}$, where each $\mathrm{C}_{60} \mathrm{H}$ is spin polarized by an intramolecular exchange and the two magnetic moments are antiferromagnetically coupled with each other. The results demonstrate that the underlying mechanism of the magnetic order in $\mathrm{fcc}_{60} \mathrm{C}_{n}$ is associated with the AFM superexchange between the magnetic moments created by $\mathrm{H}$ dopants.
\end{abstract}

DOI: 10.1103/PhysRevB.86.081415

PACS number(s): 75.10.Lp, 75.30.Hx, 75.30.Et

The observation of ferromagnetism in fullerenes has attracted intensive attention due to its interest concerning carbon magnetism and potential technological application in the emerging field of spintronics. ${ }^{1-5}$ However, there has long been controversy about whether the origin of the observed ferromagnetism is associated with extrinsic iron impurities ${ }^{6-8}$ or intrinsic defects. ${ }^{9-14}$ The latter intrinsic defects involve carbon vacancies in polymerized fullerenes ${ }^{9-11}$ or doped fullerenes ${ }^{12-14} \mathrm{C}_{60} R_{n}$ ( $R$ : nonmagnetic elements such as $\mathrm{H}$ and $\mathrm{O}$ atoms) where doping creates fullerene radical adducts with unpaired spins localized on fullerene. Experimental studies for photo-oxidated fullerenes ${ }^{3,4}$ and hydrofullerite ${ }^{5} \mathrm{C}_{60} \mathrm{H}_{24}$ observed a signal of ferromagnetism at room temperature. However, a density-functional theory (DFT) calculation ${ }^{13}$ for $\mathrm{C}_{60} \mathrm{O}$ did not support the existence of ferromagnetism, whereas a DFT calculation ${ }^{14}$ for fcc $\mathrm{C}_{60} \mathrm{H}_{n}$ predicted a strong itinerant ferromagnetism with odd-numbered $\mathrm{H}$ dopants. From their DFT calculation within the local-density approximation, Lee and Lee $(\mathrm{LL})^{14}$ found that $\mathrm{H}$ dopants on an fcc $\mathrm{C}_{60}$ crystal create quasilocalized $\pi$ electrons leading to a narrow half-filled band, and concluded that a direct overlap of the $\pi$ electrons between adjacent $\mathrm{C}_{60} \mathrm{H}_{n}$ molecules gives rise to Stoner (itinerant) ferromagnetism with an exchange splitting of $\sim 0.2 \mathrm{eV}$. However, this exchange splitting is not due to the Stoner-type FM exchange but to an intramolecular exchange (i.e., Stoner parameter I), as discussed below. As a matter of fact, the ferromagnetic (FM) and antiferromagnetic (AFM) order is governed by the exchange interaction energy between neighboring magnetic moments (i.e., exchange coupling constant $J$ in the Heisenberg model).

Recently, it was reported ${ }^{15}$ that the microscopic mechanism of defect-induced magnetism in dilute magnetic semiconductors could be similar to that in carbon-based materials such as $\mathrm{C}_{60}$ polymers, TDAE- $\mathrm{C}_{60}$, graphene ribbons, and irradiated graphite. There are two different mechanisms that describe the exchange interaction in diluted magnetic semiconductors: The double-exchange mechanism favors FM coupling, whereas the superexchange mechanism usually leads to AFM coupling. ${ }^{16}$ For the cases of double exchange and superexchange, the spin-polarized density-of-states (DOS) of transition-metal impurities in a wide-band-gap semiconductor are schematically shown in Figs. 1(a) and 1(b), respectively. In double exchange, the Fermi level $\left(E_{F}\right)$ lies in an impurity band. The width of such a partially occupied majority band can be broadened with the hopping matrix element between neighboring impurities, leading to an energy gain with the FM coupling. On the other hand, superexchange does not require a finite DOS at $E_{F}$ because it occurs via the hybridization of two impurity states which are energetically localized well below and well above $E_{F}$. Since the electronic states with the same spin direction hybridize with each other, the highest occupied (lowest unoccupied) states are shifted to lower (higher) energies, thereby stabilizing the AFM configuration. As shown in Fig. 1(c), the spin-up and spin-down DOS of the AFM (or FM) configuration in fcc $\mathrm{C}_{60} \mathrm{H}$ show the location of $E_{F}$ between two $\mathrm{H}$-induced states, corresponding to the superexchange mechanism.

In this Rapid Communication, using van der Waals energycorrected density-functional theory (DFT + vdW scheme), ${ }^{17}$ we investigate the stability of fcc $\mathrm{C}_{60} \mathrm{H}_{n}$ within the NM, FM, and AFM configurations. We find that the AFM configuration is energetically more stable than the FM one by a few meV. The charge and spin characters of fcc $\mathrm{C}_{60} \mathrm{H}_{n}$ is shown to be nearly equal to those of a $\mathrm{C}_{60} \mathrm{H}_{n}$ molecule as well as its dimer $\left(\mathrm{C}_{60} \mathrm{H}_{n}\right)_{2}$. Therefore, we conclude that the spin splitting of the $\mathrm{H}$-induced states in fcc $\mathrm{C}_{60} \mathrm{H}_{n}$ is due to the local polarization effect caused by an intramolecular exchange. The underlying mechanism of the magnetic order in fcc $\mathrm{C}_{60} \mathrm{H}_{n}$ is found to be associated with the AFM superexchange, rather than a recently proposed $^{14}$ Stoner-type FM exchange.

The present DFT + vdW calculations were performed using the FHI-aims $\operatorname{code}^{18}$ which provides for an accurate, allelectron description based on numeric atom-centered orbitals, with tight computational settings and accurate tier- 2 basis sets. This calculation scheme combined with the generalized gradient approximation (GGA) of the Perdew-Burke-Ernzerhof ${ }^{19}$ 
(a)

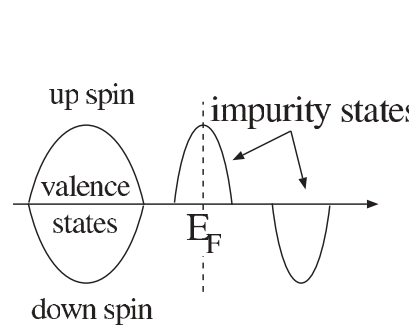

(c)

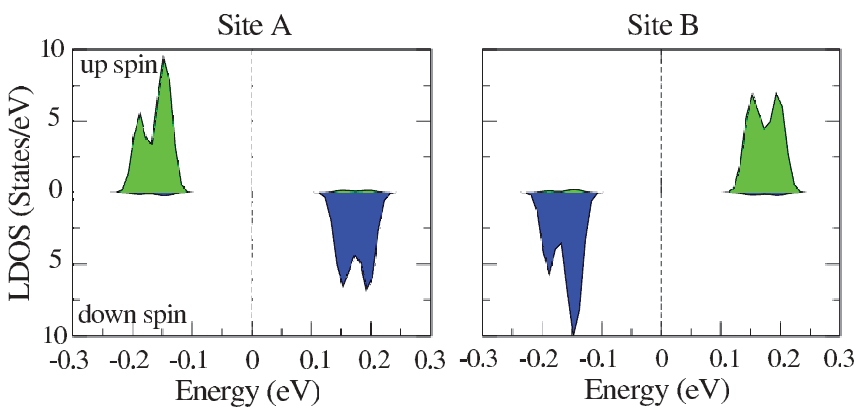

FIG. 1. (Color online) Schematic diagrams of the spin-polarized DOS in the cases of (a) double exchange and (b) superexchange. The local density of states (LDOS) obtained from the $\mathrm{AFM}_{3}-S_{3}$ configuration [see Fig. 2(b)] of fcc $\mathrm{C}_{60} \mathrm{H}$ is also given in (c). The sites $\mathrm{A}$ and $\mathrm{B}$ indicate the $\mathrm{C}_{60} \mathrm{H}$ molecules having the positive and negative magnetic moments, respectively. The LDOS are taken by summing the LDOS over the $\mathrm{C}$ atom (bonding to the hydrogenated $\mathrm{C}$ atom with the 6:6 bond) and its nearest and next nearest $C$ atoms.

is required since using the GGA functional without vdW interactions results in no visible minimum in the binding energy curve of fcc $\mathrm{C}_{60} \mathrm{H}_{n}$ as a function of the lattice constant. In order to compare the energetics of the NM, FM, and AFM configurations with the same accuracy, we employed a cubic unit cell whose volume is four, four, and two times larger than those of the primitive unit cells of the NM, FM, and AFM configurations, respectively. ${ }^{20}$ The k-space integration was done with meshes of $3 \times 3 \times 3 \mathbf{k}$ points in the Brillouin zone of the cubic unit cell. ${ }^{21}$ All the atoms were allowed to relax along the calculated forces until all the residual force components are less than $0.01 \mathrm{eV} / \AA$. For all the calculations of fcc $\mathrm{C}_{60} \mathrm{H}_{n}$, we fixed the lattice constant as $14.14 \AA$ (optimized from fcc $\mathrm{C}_{60}$ ) because $\mathrm{H}$ adsorption was found to decrease the lattice constant by less than $1 \%$. This lattice constant of fcc $\mathrm{C}_{60}$ is in good agreement with the experimental data ${ }^{5}$ of $14.17 \AA$. We note that there are two different bond lengths in fcc $\mathrm{C}_{60}$ : The 5:6 bond (constituting regular pentagons) has $1.45 \AA$, while the $6: 6$ bond (shared by two hexagons) has $1.40 \AA$. These values are close to the experimental ${ }^{22}$ data of 1.46 and $1.40 \AA$ for a $\mathrm{C}_{60}$ molecule, respectively.

We first consider the NM configuration of fcc $\mathrm{C}_{60} \mathrm{H}$ using a spin-unpolarized calculation. As shown in Fig. 2(a), there are three different adsorption sites $\left(S_{1}, S_{2}\right.$, and $\left.S_{3}\right)$ for an $\mathrm{H}$ atom on fcc $\mathrm{C}_{60}$. The relative stability of such different adsorption sites (hereafter designated as the NM- $S_{1}, \mathrm{NM}-S_{2}$, and NM- $S_{3}$ configurations) are given in Table I. We find that the (a)

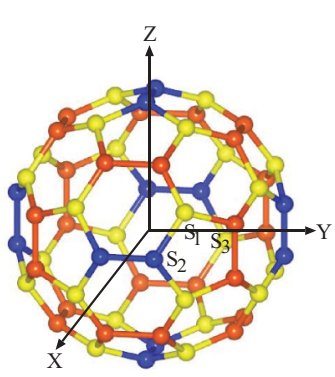

(b)

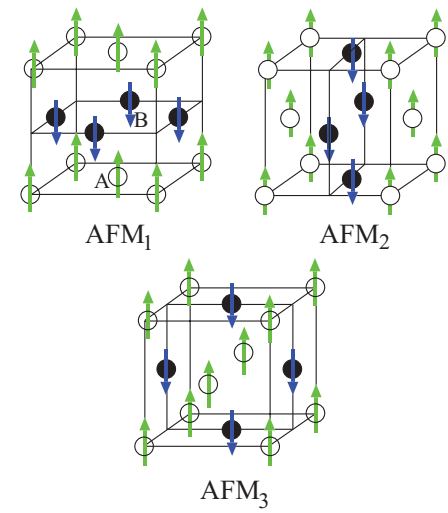

FIG. 2. (Color online) (a) Orientation of a $\mathrm{C}_{60}$ molecule in fcc $\mathrm{C}_{60} \mathrm{H}_{n}$. The light, dark, and gray colored circles represent the three different adsorption sites $S_{1}, S_{2}$, and $S_{3}$, respectively. The $\mathbf{x}, \mathbf{y}$, and $\mathbf{z}$ axes point along the [100], [010], and [001] directions, respectively. Each axis crosses the $6: 6$ bond at the midpoint. (b) Schematic diagrams of three different AFM configurations (designated as $\mathrm{AFM}_{1}$, $\mathrm{AFM}_{2}$, and $\mathrm{AFM}_{3}$ ) with AFM spin alignments along the [001], [010], and [100] directions, respectively. Here the direction of the arrow representing the up (down) spin moment is not specified along the positive (negative) $\mathbf{z}$ axis because of the neglect of spin-orbit coupling in the calculations.

NM- $S_{2}\left(\mathrm{NM}-S_{3}\right)$ configuration is more stable than NM- $S_{1}$ by 17 (84) meV. We note that an $\mathrm{H}$ adsorption on fcc $\mathrm{C}_{60}$ creates a quasilocalized $\pi$ electron distributed over the fullerene around the hydrogenated $\mathrm{C}$ atom (as discussed below), contributing to hydrogen bonding or vdW interaction between neighboring $\mathrm{C}_{60}$ molecules. Since the distance between the $\mathrm{H}$ atom and its neighboring $\mathrm{C}_{60}$ molecule is in the order of $d_{\mathrm{H}-\mathrm{C}_{60}}\left(\mathrm{NM}-S_{1}\right)>$ $d_{\mathrm{H}-\mathrm{C}_{60}}\left(\mathrm{NM}-S_{2}\right)>d_{\mathrm{H}-\mathrm{C}_{60}}\left(\mathrm{NM}-S_{3}\right)$, the stability of the three NM configurations are likely to be ordered as $E\left(\mathrm{NM}-S_{1}\right)>E(\mathrm{NM}-$ $\left.S_{2}\right)>E\left(\mathrm{NM}-S_{3}\right)$.

Figure 3(a) shows the calculated electronic band structure for the NM- $S_{3}$ configuration. It is found that a single impurity band created by an $\mathrm{H}$ adsorption crosses the Fermi level, yielding a half-filled band. The charge character of such an $\mathrm{H}$-induced state at the $\Gamma$ point is displayed in Fig. 3(a), representing a quasilocalized $\pi$ electron which is not only localized at the $\mathrm{C}$ atom (labeled as $\mathrm{C}_{0}$ ) bonding to the hydrogenated $\mathrm{C}$ atom with the $6: 6$ double bond but also distributed over the fullerene centered at the $\mathrm{C}_{0}$ atom. We note that the charge distribution of such a quasilocalized $\pi$ electron in fcc $\mathrm{C}_{60} \mathrm{H}$ is nearly equal to that of the highest occupied molecular orbital of a spin-unpolarized $\mathrm{C}_{60} \mathrm{H}$ molecule (see

TABLE I. Calculated total energies (in $\mathrm{meV} / \mathrm{C}_{60} \mathrm{H}$ ) for the NM, $\mathrm{FM}, \mathrm{AFM}_{1}, \mathrm{AFM}_{2}$, and $\mathrm{AFM}_{3}$ configurations of fcc $\mathrm{C}_{60} \mathrm{H}$ at the $S_{1}$, $S_{2}$, and $S_{3}$ sites. The energy of NM- $S_{1}$ is set to zero.

\begin{tabular}{rrrrrr}
\hline \hline & $\mathrm{NM}$ & $\mathrm{FM}$ & $\mathrm{AFM}_{1}$ & $\mathrm{AFM}_{2}$ & $\mathrm{AFM}_{3}$ \\
\hline$S_{1}$ & 0 & -47 & -50 & -49 & -46 \\
$S_{2}$ & -17 & -54 & -59 & -61 & -55 \\
$S_{3}$ & -84 & -122 & -125 & -128 & -130 \\
\hline \hline
\end{tabular}


(a)
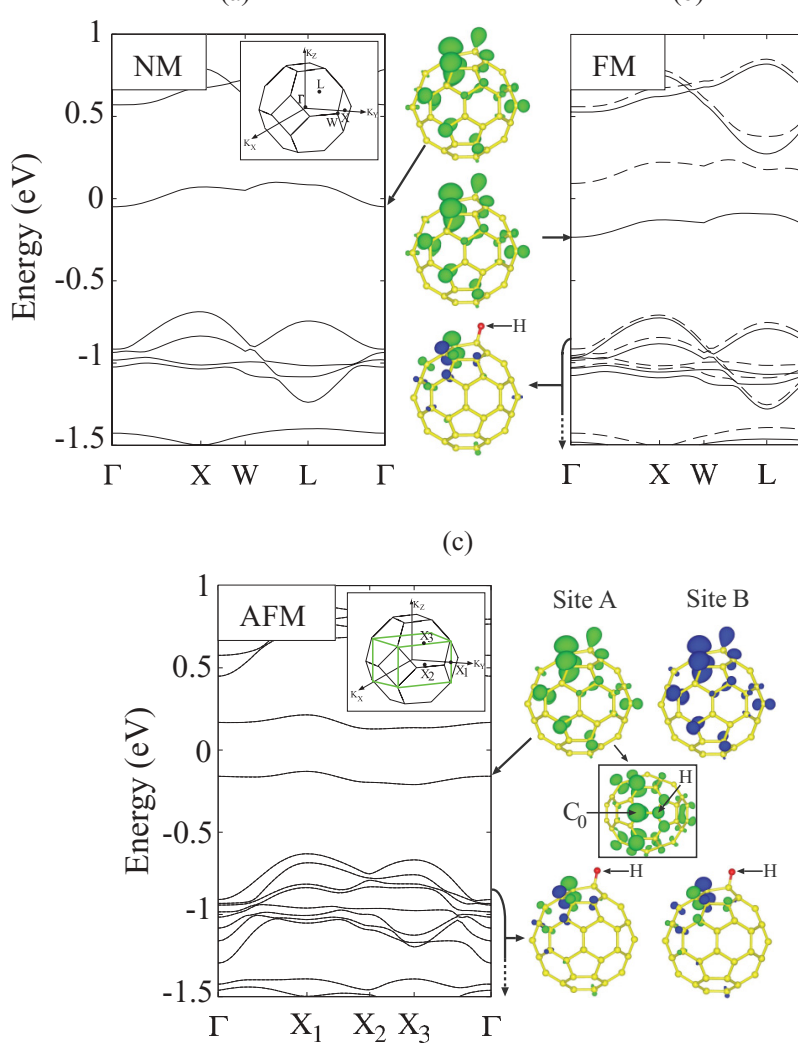

FIG. 3. (Color online) Calculated band structures for the (a) NM$S_{3}$, (b) FM- $S_{3}$, and (c) $\mathrm{AFM}_{3}-S_{3}$ configurations. In (b), the solid (dashed) lines represent the spin-up (spin-down) bands, while in (c), the spin-up and spin-down bands are degenerate. The insets in (a) and (c) show the Brillouin zones for the primitive cells of NM- $S_{3}$ (or FM- $S_{3}$ ) and $\mathrm{AFM}_{3}-S_{3}$. The energy zero represents the Fermi level. For NM- $S_{3}$, the charge character of the $\mathrm{H}$-induced state at the $\Gamma$ point is drawn with an isosurface of 0.01 electron $/ \AA^{3}$. For FM- $S_{3}$, and $\mathrm{AFM}_{3}-S_{3}$, the spin characters of the $\mathrm{H}$-induced state and the valence states are also drawn. The light (dark) colored isosuface represents the positive (negative) spin density with an isosurface of $0.01(-0.01)$ electrons $/ \AA^{3}$. The inset in (c) shows the top view of spin character at the site $\mathrm{A}$.

the Supplemental Material ${ }^{23}$ ). Thus, we can say that fcc $\mathrm{C}_{60} \mathrm{H}$ has a very weak electronic coupling between adjacent $\mathrm{C}_{60} \mathrm{H}$ molecules. Considering the fact that the ground state of a $\mathrm{C}_{60} \mathrm{H}$ molecule is spin polarized, the spin splitting of the $\mathrm{H}$-induced state is most likely to occur via an intramolecular exchange rather than the recently proposed $^{14}$ Stoner-type exchange interaction of itinerant electrons between adjacent $\mathrm{C}_{60} \mathrm{H}$ molecules.

Next, we perform spin-polarized calculations for fcc $\mathrm{C}_{60} \mathrm{H}$ within the FM and AFM configurations. For the AFM configuration, we consider three different AFM spin alignments along the [001], [010], and [100] directions [see Fig. 2(b), hereafter designated as $\mathrm{AFM}_{1}, \mathrm{AFM}_{2}$, and $\mathrm{AFM}_{3}$, respectively]. This AFM configuration showing the AFM coupling of twodimensional FM layers along the three directions is similar to that in layered magnetic materials. ${ }^{24}$ The calculated total energies of these FM and AFM configurations relative to the NM- $S_{1}$ configuration are given in Table I. For the adsorption sites of $S_{1}, S_{2}$, and $S_{3}$, the FM (AFM) configuration is found to be more stable than the NM one by 47 (46-50), 37 (38-44), and 38 (41-46) meV, respectively, showing that the AFM ordering is preferred over the FM one. ${ }^{25}$ From the energy difference between the AFM and FM configurations, ${ }^{26}$ we obtain the exchange coupling constant $J$ between neighboring magnetic moments as a few meV. This weak AFM exchange coupling in fcc $\mathrm{C}_{60} \mathrm{H}$ contrasts with a recent DFT study ${ }^{14}$ which proposed a strong Stoner-type FM exchange interaction energy of $\sim 0.2 \mathrm{eV}$ by using the energy difference between the NM and FM configurations. Using the mean field approximation, ${ }^{27}$ we estimate a transition temperature of fcc $\mathrm{C}_{60} \mathrm{H}$ from the AFM to the paramagnetic phase as $\sim 30 \mathrm{~K}$, contrasting with an experimental observation ${ }^{5}$ of room-temperature ferromagnetism in hydrofullerite $\mathrm{C}_{60} \mathrm{H}_{24}$. Here the ferromagnetic signal of $\mathrm{C}_{60} \mathrm{H}_{24}$ was observed under the high hydrogen pressures of 0.6 and $3 \mathrm{GPa}$, but gradually decreased at ambient conditions: Moreover, the magnetization values were not well reproduced at the sample synthesis. ${ }^{5}$ We note that the Hubbard correlation energy $U$ for fcc $\mathrm{C}_{60}$ was measured to be about $1.5 \mathrm{eV}$ and those of alkali fullerides such as $\mathrm{RbC}_{60}, \mathrm{~K}_{3} \mathrm{C}_{60}$, and $\mathrm{Cs}_{4} \mathrm{C}_{60}$ ranged from 0.7 to $1 \mathrm{eV}^{28,29}$ Assuming that the $U$ value for fcc $\mathrm{C}_{60} \mathrm{H}_{n}$ is similar to those of alkali fullerides, we can estimate a large ratio of $U / t$ with an electron hopping parameter $(t)$ of $\sim 0.1 \mathrm{eV}$, estimated from the bandwidth of the $\mathrm{H}$-induced state (see Fig. 3). The large value of $U / t$ gives rise to an AFM ground state from the Hubbard Hamiltonian, ${ }^{30}$ consistent with our DFT + vdW result.

Figures 3(b) and 3(c) show the electronic band structures for the FM- $S_{3}$ and $\mathrm{AFM}_{3}-S_{3}$ configurations, respectively. We find that the $\mathrm{AFM}_{3}-S_{3}$ configuration has a band gap of $0.26 \mathrm{eV}$, larger than that $(0.18 \mathrm{eV})$ of $\mathrm{FM}-S_{3}$. This manifests that the electronic energy gain is relatively enhanced by the AFM spin ordering, compared to the FM one. The spin characters of the $\mathrm{H}$-induced state as well as the valence states in the FM- $S_{3}$ and $\mathrm{AFM}_{3}-S_{3}$ configurations are also displayed in Figs. 3(b) and 3 (c), respectively. It is found that the spin distributions of the $\mathrm{H}$ induced and valence states in FM- $S_{3}$ are almost equal to those in the A site of $\mathrm{AFM}_{3}-S_{3}$ (see the Supplemental Material ${ }^{23}$ ). Such indifference of the spin characters between FM- $S_{3}$ and $\mathrm{AFM}_{3}-S_{3}$ results in the above-mentioned very weak exchange coupling in $\mathrm{fcc}_{\mathrm{C}} \mathrm{C}_{60} \mathrm{H}$.

It is noteworthy that a recent DFT calculation ${ }^{31}$ for the $\mathrm{H}$ defects in graphene predicted the presence of a strong itinerant (Stoner) magnetism, where the coupling between the magnetic moments is either FM or AFM, depending on whether $\mathrm{H}$ atoms adsorb on the same or different graphene sublattices, respectively. This strong coupling mechanism is not only due to the bipartite nature of the graphene lattice which gives rise to a long-range spin polarization of the defect states, but also to the exchange spin-polarization effect in the valence states (i.e., the response of the fully populated valence states to the magnetization of the defect states). These two features are also present in fcc $\mathrm{C}_{60} \mathrm{H}$, showing (i) the spin polarization of the $\mathrm{H}$-induced state which is distributed over the fullerene centered at the $\mathrm{C}_{0}$ atom [see the inset in Fig. 3(c)] and (ii) the spin polarization of valence states [see Figs. 3(b) and 3(c)]. However, we have to emphasize that, unlike the case of graphene ${ }^{31}$ where the H-induced magnetic moments are ferromagnetically coupled through a 


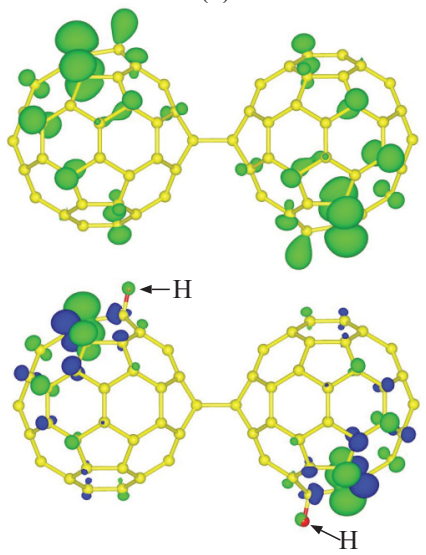

(b)

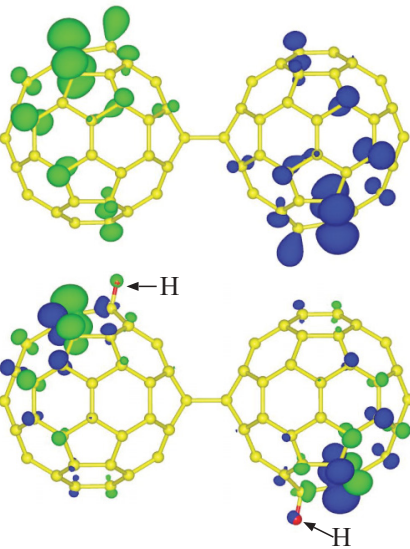

FIG. 4. (Color online) Calculated spin characters of the HOMO (upper panel) and the occupied states excluding the HOMO (lower panel) for a dimer $\left(\mathrm{C}_{60} \mathrm{H}\right)_{2}$ within (a) the $\mathrm{FM}$ configuration and (b) the AFM configuration. The light (dark) colored isosuface represents the positive (negative) spin density with an isosurface of $0.01(-0.01)$ electrons $/ \AA^{3}$.

direct overlap of the $\pi$ electrons, fcc $\mathrm{C}_{60} \mathrm{H}$ cannot have such a direct overlap of the $\mathrm{H}$-induced states because of a large separation between adjacent $\mathrm{C}_{60} \mathrm{H}$ molecules. We note that the LDOS [Fig. 1(c)] of the $\mathrm{AFM}_{3}-S_{3}$ configuration show no electronic states between the occupied and unoccupied $\mathrm{H}$-induced states, therefore leading to the AFM coupling via the superexchange mechanism. ${ }^{16}$

The preference of AFM over FM coupling also exists in a dimer $\left(\mathrm{C}_{60} \mathrm{H}\right)_{2}$ where the $\mathrm{C}-\mathrm{C}$ bond length between two $\mathrm{C}_{60} \mathrm{H}$ molecules is calculated to be $1.59 \AA$. We find that the FM and AFM configurations are more stable than the NM one by 62 and $65 \mathrm{meV}$, respectively. The energy difference between the AFM and FM configurations gives rise to $J=-3 \mathrm{meV}$, comparable with those (see Table I) in fcc $\mathrm{C}_{60} \mathrm{H}$. However, a previous DFT calculation $^{12}$ for $\left(\mathrm{C}_{60} \mathrm{H}\right)_{2}$ incorrectly estimated the exchange coupling constant as $J=0.58 \mathrm{eV}$ from the energy difference between the NM and FM configurations. We note that the separation between the two fullerenes in a $\mathrm{C}_{60}$ dimer is much shorter than that (3.11 $\AA$ ) in fcc $\mathrm{C}_{60}$, but the number of adjacent fullerences in the former decreases by a factor of 12 compared to the latter. Figures 4(a) and 4(b) show the spin densities of the highest occupied molecular orbital (HOMO) and the occupied states excluding the HOMO for the FM and AFM configurations of $\left(\mathrm{C}_{60} \mathrm{H}\right)_{2}$, respectively. These spin characters in $\left(\mathrm{C}_{60} \mathrm{H}\right)_{2}$ are nearly the same as those in an isolated $\mathrm{C}_{60} \mathrm{H}$ molecule as well as fcc $\mathrm{C}_{60} \mathrm{H}$. Thus, an individual molecule in $\left(\mathrm{C}_{60} \mathrm{H}\right)_{2}$ and fcc $\mathrm{C}_{60} \mathrm{H}$ has the same magnetic moment as that $\left(1 \mu_{\mathrm{B}}\right)$ of an isolated $\mathrm{C}_{60} \mathrm{H}$ molecule.

According to a recent DFT calculation, ${ }^{14}$ the ground state of fcc $\mathrm{C}_{60} \mathrm{H}_{n}$ with even (odd)-numbered $n$ was predicted to be NM (FM) without considering the AFM configuration. For fcc $\mathrm{C}_{60} \mathrm{H}_{2}$, using a previously ${ }^{14}$ obtained structure which contains two $\mathrm{H}$ atoms bonded to opposite sides of the $6: 6$ double bond, we find that the two adsorbed $\mathrm{H}$ atoms do not create quasilocalized $\pi$ electrons, leading to the NM ground state. On the other hand, we find that fcc $\mathrm{C}_{60} \mathrm{H}_{3}$, whose stable structure contains two $\mathrm{H}$ atoms bonded to opposite sides of the 6:6 double bond together with one $\mathrm{H}$ atom adsorbed at the nearest-neighbor sites, ${ }^{14}$ creates quasilocalized $\pi$ electrons. The calculated electronic structures for the FM and AFM configurations of fcc $\mathrm{C}_{60} \mathrm{H}_{3}$ are similar to the corresponding ones of fcc $\mathrm{C}_{60} \mathrm{H}$ (see the Supplemental Material ${ }^{23}$ ). As a result, fcc $\mathrm{C}_{60} \mathrm{H}_{3}$ is found to favor the AFM configuration over the FM one by $\sim 10 \mathrm{meV}$, slightly larger than the case of fcc $\mathrm{C}_{60} \mathrm{H}$.

In summary, using van der Waals energy-corrected densityfunctional theory, we have studied the magnetic order of fcc $\mathrm{C}_{60} \mathrm{H}_{n}$ (for $n=$ odd) within the FM and AFM configurations. We found that the AFM configuration is slightly more stable than the FM one by a few meV. The charge and spin characters of fcc $\mathrm{C}_{60} \mathrm{H}_{n}$ were shown to be nearly equal to those of a $\mathrm{C}_{60} \mathrm{H}_{n}$ molecule and a $\left(\mathrm{C}_{60} \mathrm{H}_{n}\right)_{2}$ dimer. Therefore, we concluded that an individual molecule in fcc $\mathrm{C}_{60} \mathrm{H}_{n}$ is spin polarized by an intramolecular exchange, and that such magnetic moments are antiferromangnetically coupled with each other via the superexchange mechanism.

This work was supported by National Research Foundation of Korea (NRF) grant funded by the Korean Government (NRF-2011-0015754). The calculations were performed by KISTI supercomputing center through the strategic support program (KSC-2011-C3-08) for the supercomputing application research.
*Corresponding author: chojh@ hanyang.ac.kr

${ }^{1}$ T. L. Makarova, B. Sundqvist, R. Höhne, P. Esquinazi, Y. Kopelevich, P. Scharff, V. A. Davydov, L. S. Kashevarova, and A. V. Rakhmanina, Nature (London) 413, 716 (2001).

${ }^{2}$ P. M. Allemand, K. C. Khemani, A. Koch, F. Wudl, K. Holczer, S. Donovan, G. Gruner, and J. D. Thompson, Science 253, 301 (1991).

${ }^{3}$ T. L. Makarova, K. H. Han, P. Esquinazi, R. R. da Silva, Y. Kopelevich, I. B. Zakharova, and B. Sundqvist, Carbon 41, 1575 (2003).

${ }^{4}$ F. J. Owens, Z. Iqbal, L. Belova, and K. V. Rao, Phys. Rev. B 69, 033403 (2004).
${ }^{5}$ V. E. Antonov, I. O. Bashkin, S. S. Khasanov, A. P. Moravsky, Y. G. Morozov, Y. M. Shulga, Y. A. Ossipyan, and E. G. Ponyatovsky, J. Alloys Compd. 330-332, 365 (2002).

${ }^{6}$ R. Höhne and P. Esquinazi, Adv. Mater. 14, 753 (2002).

${ }^{7}$ A. Talyzin, A. Dzwilewski, L. Dubrovinsky, A. Setzer, and P. Esquinazi, Eur. Phys. J. B: Complex Syst. 55, 57 (2007).

${ }^{8}$ T. L. Makarova, B. Sundqvist, R. Höhne, P. Esquinazi, Y. Kopelevich, P. Scharff, V. Davydov, L. S. Kashevarova, and A. V. Rakhmanina, Nature (London) 440, 707 (2006).

${ }^{9}$ J. A. Chan, B. Montanari, W. L. Chan, and N. M. Harrison, Mol. Phys. 103, 2573 (2005). 
${ }^{10}$ J. A. Chan, B. Montanari, J. D. Gale, S. M. Bennington, J. W. Taylor, and N. M. Harrison, Phys. Rev. B 70, 041403 (2004).

${ }^{11}$ D. W. Boukhvalov and M. I. Katsnelson, Eur. Phys. J. B: Complex Syst. 68, 529 (2009).

${ }^{12}$ O. E. Kvyatkovskii, I. B. Zakharova, A. L. Shelankov, and T. L. Makarova, Phys. Rev. B 72, 214426 (2005).

${ }^{13}$ O. E. Kvyatkovskii, and I. B. Zakharova, Fullerences, Nanotubes Carbon Nanostruct. 16, 574 (2008).

${ }^{14}$ K. W. Lee and C. E. Lee, Phys. Rev. Lett. 106, 166402 (2011).

${ }^{15}$ A. N. Andriotis, R. M. Sheetz, and M. Madhu, J. Phys.: Condens. Matter 22, 334210 (2010); A. N. Andriotis, M. Menon, R. M. Sheetz, and L. Chernozatonskii, Phys. Rev. Lett. 90, 026801 (2003).

${ }^{16}$ K. Sato, L. Bergqvist, J. Kudrnovsky, P. H. Dederichs, O. Eriksson, I. Turek, B. Sanyal, G. Bouzerar, H. Katayama-Yoshida, V. A. Dinh, T. Fukushima, H. Kizaki, and R. Zeller, Rev. Mod. Phys. 82, 1633 (2010).

${ }^{17}$ A. Tkatchenko and M. Scheffler, Phys. Rev. Lett. 102, 073005 (2009).

${ }^{18}$ V. Blum, R. Gehrke, F. Hanke, P. Havu, V. Havu, X. Ren, K. Reuter, and M. Scheffler, Comput. Phys. Commun. 180, 2175 (2009).

${ }^{19}$ J. P. Perdew, K. Burke, and M. Ernzerhof, Phys. Rev. Lett. 77, 3865 (1996).

${ }^{20} \mathrm{We}$ assume that four fullerenes in the cubic unit cell have the same orientation. This assumption was also employed in the previous study of Lee and Lee (Ref. 14).

${ }^{21}$ The used $3 \times 3 \times 3 \mathbf{k}$ points in the Brillouin zone of the cubic unit cell are sufficiently enough to give converged results for the geometric and magnetic properties of fcc $\mathrm{C}_{60} \mathrm{H}_{n}$. This is a denser k-point sampling compared to the previously (Ref. 14) employed $4 \times 4 \times 4 \mathbf{k}$ points in the Brillouin zone for the fcc unit cell.

${ }^{22}$ K. Hedberg, L. Hedberg, D. S. Bethune, C. A. Brown, H. C. Dorh, R. D. Johnson, and M. de Vrie, Science 254, 410 (1991).

${ }^{23}$ See Supplemental Material at http://link.aps.org/supplemental/ 10.1103/PhysRevB.86.081415 for the charge density of the HOMO state for a spin-unpolarized $\mathrm{C}_{60} \mathrm{H}$ molecule, the total spin densities of the FM-S $\mathrm{S}_{3}$, and $\mathrm{AFM}_{3}-\mathrm{S}_{3}$ configurations of fcc $\mathrm{C}_{60} \mathrm{H}$, and the band structures for the FM, and AFM configurations of fcc $\mathrm{C}_{60} \mathrm{H}_{3}$.

${ }^{24}$ M. G. Samant et al., Phys. Rev. Lett. 72, 1112 (1994).

${ }^{25}$ The GGA calculation for the structure optimized by the GGA + vdW scheme changes little the energy difference between the FM and AFM configurations by less than $1 \mathrm{meV}$. Thus, we can say that the inclusion of $\mathrm{vdW}$ interactions contributes to binding of fcc $\mathrm{C}_{60} \mathrm{H}_{n}$, but does not affect the energy difference between the FM and AFM configurations.

${ }^{26}$ L. Noodleman, J. Chem. Phys. 74, 5737 (1981); L. Noodleman and E. R. Davidson, Chem. Phys. 109, 131 (1986).

${ }^{27}$ G. S. Rushbrooke and P. J. Wood, Mol. Phys. 1, 257 (1958); 6, 409 (1963).

${ }^{28}$ O. Gunnarsson, Rev. Mod. Phys. 69, 575 (1997).

${ }^{29}$ R. Macovez, M. R. C. Hunt, A. Goldoni, M. Pedio, and P. Rudolf, J. Electron Spectrosc. Relat. Phenom. 183, 94 (2011).

${ }^{30}$ B. Alvarez-Fernández and J. A. Blanco, Eur. J. Phys. 23, 11 (2002).

${ }^{31}$ O. V. Yazyev and L. Helm, Phys. Rev. B 75, 125408 (2007). 